IJMMS 31:8 (2002) 451-461

PII. S0161171202012735

http://ijmms.hindawi.com

(c) Hindawi Publishing Corp.

\title{
A SECOND-ORDER IMPULSIVE CAUCHY PROBLEM
}

\section{EDUARDO HERNÁNDEZ MORALES}

Received 11 April 2001 and in revised form 20 December 2001

We study the existence of mild and classical solutions for an abstract second-order impulsive Cauchy problem modeled in the form $\ddot{u}(t)=A u(t)+f(t, u(t), \dot{u}(t)), t \in\left(-T_{0}, T_{1}\right)$, $t \neq t_{i} ; u(0)=x_{0}, \dot{u}(0)=y_{0} ; \Delta u\left(t_{i}\right)=I_{i}^{1}\left(u\left(t_{i}\right)\right), \Delta \dot{u}\left(t_{i}\right)=I_{i}^{2}\left(\dot{u}\left(t_{i}^{+}\right)\right)$, where $A$ is the infinitesimal generator of a strongly continuous cosine family of linear operators on a Banach space $X$ and $f, I_{i}^{1}, I_{i}^{2}$ are appropriate continuous functions.

2000 Mathematics Subject Classification: 34G20, 34A37, 47D09.

1. Introduction. This paper is concerned with the second-order impulsive Cauchy problem

$$
\begin{gathered}
\ddot{u}(t)=A u(t)+f(t, u(t), \dot{u}(t)), \quad t \in\left(-T_{0}, T_{1}\right), t \neq t_{i}, \\
u(0)=x_{0}, \quad \dot{u}(0)=y_{0}, \\
\triangle u\left(t_{i}\right)=I_{i}^{1}\left(u\left(t_{i}\right)\right), \quad \triangle \dot{u}\left(t_{i}\right)=I_{i}^{2}\left(\dot{u}\left(t_{i}^{+}\right)\right) .
\end{gathered}
$$

In problem (1.1), $A$ is the infinitesimal generator of a strongly continuous cosine family of linear operators $(C(t))_{t \in \mathbb{R}}$ on a Banach space $X$; $-T_{0}<0<t_{1}<t_{2}<\cdots<t_{n}<T_{1}$; $\triangle u\left(t_{i}\right)=u\left(t_{i}^{+}\right)-u\left(t_{i}^{-}\right), \triangle \dot{u}\left(t_{i}\right)=\dot{u}\left(t_{i}^{+}\right)-\dot{u}\left(t_{i}^{-}\right)$, and $I_{i}^{j}: X \rightarrow X, f: \mathbb{R} \times X \times X \rightarrow X$ are appropriate continuous functions.

The theory of impulsive differential equations has become an important area of investigation in recent years. Relative to this theory, we only refer the reader to the works of Rogovchenko [6, 7], Liu [5], Sun [3], and Cabada [1].

Motivated for numerous applications, recently, Liu [5] studied the first-order impulsive evolution problem

$$
\begin{gathered}
\dot{u}(t)=A u(t)+f(t, u(t)), \\
u(0)=x_{0}, \\
\triangle u\left(t_{i}\right)=I_{i}\left(u\left(t_{i}\right)\right),
\end{gathered}
$$

where $A$ is the infinitesimal generator of a $C_{0}$-semigroup of linear operators on a Banach space $X$. In the cited paper, Liu apply the semigroup theory to prove the existence of mild, strong and classical solutions for system (1.2) using usual assumptions on the function $f$.

Our goal is to give some existence results of mild and classical solutions for the second-order impulsive Cauchy problem (1.1) using the cosine functions theory. It is well known that, in general, the second-order abstract Cauchy problem

$$
\begin{gathered}
\ddot{u}(t)=A u(t), \\
u(0)=x_{0}, \quad \dot{u}(0)=y_{0},
\end{gathered}
$$


cannot be studied by reducing this system to a first-order linear equation, therefore, the existence results in this work are not consequence of those in Liu [5].

Throughout this paper, $X$ denotes an abstract Banach space endowed with a norm $\|\cdot\|$ and $C(t)$ denotes a strongly continuous operator cosine function defined on $X$ with infinitesimal generator $A$. We refer the reader to $[2,10]$ for the necessary concepts about cosine functions. Next, we only mention a few results and notations needed to establish our results. We denote by $S(t)$ the sine function associated with $C(t)$ which is defined by

$$
S(t) x:=\int_{0}^{t} C(s) x d s, \quad x \in X, t \in \mathbb{R} .
$$

For a closed operator $B: D(B) \rightarrow X$, we denote by $[D(B)]$ the space $D(B)$ endowed with the graph norm $\|\cdot\|_{B}$, that is,

$$
\|x\|_{B}=\|x\|+\|B x\|, \quad x \in D(B) .
$$

In particular, $[D(A)]$ is the space

$$
D(A)=\{x \in X: C(t) x \text { is twice continuously differentiable }\} \text {, }
$$

endowed with the norm $\|x\|_{A}=\|x\|+\|A x\|, x \in D(A)$,

Moreover, in this paper the notation $E$ stands for the space formed by the vectors $x \in X$ for which the function $C(\cdot) x$ is of class $C^{1}$. It was proved by Kisyński [4] that $E$ endowed with the norm

$$
\|x\|_{1}=\|x\|+\sup _{0 \leq t \leq 1}\|A S(t) x\|, \quad x \in E,
$$

is a Banach space. The operator-valued function $G(t)=\left[\begin{array}{cc}C(t) & S(t) \\ A S(t) & C(t)\end{array}\right]$ is a strongly continuous group of linear operators on the space $E \times X$ generated by the operator $\mathscr{A}=\left[\begin{array}{cc}0 & I \\ A & 0\end{array}\right]$ defined on $D(A) \times E$. From this, it follows that $A S(t): E \rightarrow X$ is a bounded linear operator and that $A S(t) x \rightarrow 0, t \rightarrow 0$, for each $x \in E$. Furthermore, if $x:[0, \infty) \rightarrow X$ is a locally integrable function, then

$$
y(t)=\int_{0}^{t} S(t-s) x(s) d s
$$

defines an $E$-valued continuous function. This is an immediate consequence of the fact that

$$
\int_{0}^{t} G(t-s)\left[\begin{array}{c}
0 \\
x(s)
\end{array}\right] d s=\left[\begin{array}{l}
\int_{0}^{t} S(t-s) x(s) d s \\
\int_{0}^{t} C(t-s) x(s) d s
\end{array}\right]
$$

defines an $(E \times X)$-valued continuous function.

The existence of solutions of the second-order abstract Cauchy problem

$$
\begin{gathered}
\ddot{x}(t)=A x(t)+h(t), \quad 0 \leq t \leq a, \\
x(0)=x_{0}, \quad \dot{x}(0)=x_{1},
\end{gathered}
$$


where $h:[0, a] \rightarrow X$ is an integrable function, has been discussed in [8]. Similarly, the existence of solutions of the semilinear second-order abstract Cauchy problem treated in [9]. We only mention here that the function $x(\cdot)$ given by

$$
x(t)=C(t) x_{0}+S(t) x_{1}+\int_{0}^{t} S(t-s) h(s) d s, \quad 0 \leq t \leq a
$$

is called a mild solution of (1.10). In the case in which $x_{0} \in E, x(\cdot)$ is continuously differentiable and

$$
\dot{x}=A S(t) x_{0}+C(t) x_{1}+\int_{0}^{t} C(t-s) h(s) d s .
$$

The properties in the next result are well known (see [9]).

LEMMA 1.1. In the previous condition, the following properties hold:

(1) if $x \in X$, then $S(t) x \in E$ for every $t \in \mathbb{R}$;

(2) if $x \in E$, then $S(t) x \in D(A),(d / d t) C(t) x=A S(t) x$ and $\left(d^{2} / d t^{2}\right) S(t) x=$ $A S(t) x$ for every $t \in \mathbb{R}$;

(3) if $x \in D(A)$, then $C(t) x \in D(A)$ and $\left(d^{2} / d t^{2}\right) C(t) x=A C(t) x=C(t) A x$ for every $t \in \mathbb{R}$;

(4) if $x \in D(A)$, then $S(t) x \in D(A)$ and $\left(d^{2} / d t^{2}\right) S(t) x=A S(t) x=S(t) A x$ for every $t \in \mathbb{R}$.

In Section 2, we discuss the existence of solution for the impulsive problem (1.1). Firstly, we introduce the concept of mild and classical solution for the impulsive problem (1.1) and subsequently, employing the contraction mapping principle and the ideas in Travis [10], we prove the existence of mild and classical solutions.

The terminology and notations are those generally used in operator theory. In particular, if $Z$ and $Y$ are Banach spaces, we indicate by $\mathscr{L}(Z: Y)$ the Banach space of the bounded linear operators from $Z$ into $Y$ and we abbreviate to $\mathscr{L}(Z)$ whenever $Y=Z$. In addition, $B_{r}(x: Z)$ will denote the closed ball in $Z$ with center at $x$ and radius $r$.

For a nonnegative bounded function $\xi:\left(-T_{0}, T_{1}\right] \rightarrow \mathbb{R}$ and $-T_{0}<t \leq T_{1}$, we employ the notation $\xi_{t}$ for

$$
\xi_{t}=\sup \left\{\xi(\theta): \theta \in\left(-T_{0}, t\right]\right\}
$$

Considering the notation in Liu [5], $P C_{b}\left(\left(-T_{0}, T_{1}\right): X\right)$ will be the space of the bounded functions $u:\left(-T_{0}, T_{1}\right) \rightarrow X$ such that: $u$ is continuous in $t \neq t_{i}, u$ is left continuous in each $t_{i}$ and $u\left(t_{i}^{+}\right)$exist. We always assume $P C_{b}\left(\left(-T_{0}, T_{1}\right): X\right)$ endowed with the uniform convergence norm, $\|u\|=\|u\|_{T_{1}}$.

Finally, $\mathbf{P} C_{b}^{1}\left(\left(-T_{0}, T_{1}\right): X\right)$ will be the space

$$
\begin{aligned}
& \mathbf{P} C_{b}^{1}\left(\left(-T_{0}, T_{1}\right): X\right) \\
& \quad=\left\{(u, w): u, w \in P C_{b}\left(\left(-T_{0}, T_{1}\right): X\right) \text { and } \dot{u}(t)=w(t) \text { for } t \neq t_{i}\right\}
\end{aligned}
$$

provided with the norm $\||(u, w)|\|=\|u\|_{T_{1}}+\|w\|_{T_{1}}$. 
2. Existence results. In this section, we discuss the existence of mild and classical solutions for the impulsive initial value problem (1.1). By comparison with the secondorder abstract Cauchy problem, we introduce the following definitions.

Definition 2.1. A function $(u, v) \in \mathbf{P} C_{b}^{1}\left(\left(-T_{0}, T_{1}\right): X\right)$ is a mild solution of the impulsive problem (1.1) if the impulsive conditions in (1.1) are satisfied and

$$
\begin{aligned}
u(t)= & C(t) x_{0}+S(t) y_{0}+\int_{0}^{t} S(t-s) f(s, u(s), \dot{u}(s)) d s \\
& +\sum_{0<t_{i}<t} C\left(t-t_{i}\right) I_{i}^{1}\left(u\left(t_{i}\right)\right)+\sum_{0<t_{i}<t} S\left(t-t_{i}\right) I_{i}^{2}\left(\dot{u}\left(t_{i}^{+}\right)\right),
\end{aligned}
$$

for every $t \in\left(-T_{0}, T_{1}\right)$.

Definition 2.2. A function $(u, v) \in \mathbf{P} C_{b}^{1}\left(\left(-T_{0}, T_{1}\right)\right)$ is a classical solution of the impulsive problem (1.1) if $u \in C^{2}\left(\left(-T_{0}, T_{1}\right) \backslash\left\{t_{1}, t_{2}, \ldots, t_{n}\right\}: X\right)$ and (1.1) is satisfied.

2.1. Mild solutions. In order to establish the existence of mild solutions, we introduce the following technical assumptions:

(A1) $f: \mathbb{R} \times X \times X \rightarrow X$ is a continuous function and there exist positive constants $K^{1}(f)$ and $K^{2}(f)$ such that

$$
\left\|f(t, x, y)-f\left(t, x^{\prime}, y^{\prime}\right)\right\| \leq K^{1}(f)\left\|x-x^{\prime}\right\|+K^{2}(f)\left\|y-y^{\prime}\right\|
$$

for every $x, x^{\prime}, y$ and $y^{\prime} \in X$;

(A2) the functions $I_{i}^{j}: X \rightarrow X$ are continuous and there exist positive constants $K\left(I_{i}^{j}\right)$ such that

$$
\left\|I_{i}^{j}(x)-I_{i}^{j}\left(x^{\prime}\right)\right\| \leq K\left(I_{i}^{j}\right)\left\|x-x^{\prime}\right\|,
$$

for every $x, x^{\prime} \in X$

(A3) there exist functions $B:\left[-T_{0}, T_{1}\right] \rightarrow \mathscr{L}(X), F_{i}: X \rightarrow X, i \in\{1,2, \ldots, n\}$, such that:

(i) $B(0)=0, B(\cdot)$ is strongly continuous and $(d / d t) C(t) I_{i}^{1}(x)=B(t) F_{i}(x)$ for every $x \in X$ and $i \in\{1,2, \ldots, n\}$

(ii) for each $i \in\{1,2, \ldots, n\}$, exists a positive constant $K\left(F_{i}\right)$ such that

$$
\left\|F_{i}(x)-F_{i}\left(x^{\prime}\right)\right\| \leq K\left(F_{i}\right)\left\|x-x^{\prime}\right\|, \quad \forall x, x^{\prime} \in X .
$$

THEOREM 2.3. Let $x_{0} \in E, y_{0} \in X$ and assume that assumptions (A1)-(A3) hold. If $\max \left\{\Lambda_{1}, \Lambda_{2}\right\}<1$, where

$$
\begin{aligned}
& \Lambda_{1}=K^{1}(f)\left(\|S\|_{T_{1}}+\|C\|_{T_{1}}\right)\left(T_{0}+T_{1}\right)+\sum_{i=1}^{n}\left(\|C\|_{T_{1}} K\left(I_{i}^{1}\right)+\|B\|_{T_{1}} K\left(F_{i}\right)\right), \\
& \Lambda_{2}=\left(\|S\|_{T_{1}}+\|C\|_{T_{1}}\right)\left(K^{2}(f)\left(T_{0}+T_{1}\right)+\sum_{i=1}^{n} K\left(I_{i}^{2}\right)\right),
\end{aligned}
$$

then there exists a unique mild solution of the impulsive problem (1.1). 
Proof. For $(u, w) \in \mathbf{P} C_{b}^{1}\left(\left(-T_{0}, T_{1}\right): X\right)$, we define $\Phi(u, w)=\left(\Phi_{1}(u, w), \Phi_{2}(u, w)\right)$, where

$$
\begin{aligned}
\Phi_{1}(u, w)(t)= & C(t) x_{0}+S(t) y_{0}+\int_{0}^{t} S(t-s) f(s, u(s), \dot{u}(s)) d s \\
& +\sum_{0<t_{i}<t} C\left(t-t_{i}\right) I_{i}^{1}\left(u\left(t_{i}\right)\right)+\sum_{0<t_{i}<t} S\left(t-t_{i}\right) I_{i}^{2}\left(\dot{u}\left(t_{i}^{+}\right)\right), \\
\Phi_{2}(u, w)(t)= & A S(t) x_{0}+C(t) y_{0}+\int_{0}^{t} C(t-s) f(s, u(s), \dot{u}(s)) d s \\
& +\sum_{0<t_{i}<t} B\left(t-t_{i}\right) F_{i}\left(u\left(t_{i}\right)\right)+\sum_{0<t_{i}<t} C\left(t-t_{i}\right) I_{i}^{2}\left(\dot{u}\left(t_{i}^{+}\right)\right) .
\end{aligned}
$$

Clearly, $\Phi(u, w) \in \mathscr{P} C_{b}^{1}\left(\left(-T_{0}, T_{1}\right): X\right)$. In order to prove that $\Phi$ is a contraction mapping on $\mathbf{P} C_{b}^{1}\left(\left(-T_{0}, T_{1}\right): X\right)$, we take $(u, w),(v, z)$ in $\mathbf{P} C_{b}^{1}\left(\left(-T_{0}, T_{1}\right): X\right)$. From assumption (A1), we see that for $t \in\left(-T_{0}, T_{1}\right)$

$$
\begin{aligned}
\left\|\Phi_{1}(u, w)(t)-\Phi_{1}(v, z)(t)\right\| \leq & \int_{0}^{t}\|S\|_{T_{1}}\left(K^{1}(f)\|u-v\|_{\theta}+K^{2}(f)\|\dot{u}-\dot{v}\|_{\theta}\right) d \theta \\
& +\sum_{0<t_{i}<t}\|C\|_{T_{1}} K\left(I_{i}^{1}\right)\left\|u\left(t_{i}\right)-v\left(t_{i}\right)\right\| \\
& +\sum_{0<t_{i}<t}\|S\|_{T_{1}} K\left(I_{i}^{2}\right)\left\|\dot{u}\left(t_{i}^{+}\right)-\dot{v}\left(t_{i}^{+}\right)\right\|,
\end{aligned}
$$

thus

$$
\begin{aligned}
\left\|\Phi_{1}(u, w)-\Phi_{1}(v, z)\right\|_{T_{1}} \leq & \left(\|S\|_{T_{1}} K^{1}(f)\left(T_{0}+T_{1}\right)+\sum_{i=1}^{n}\|C\|_{T_{1}} K\left(I_{i}^{1}\right)\right)\|u-v\|_{T_{1}} \\
& +\|S\|_{T_{1}}\left(K^{2}(f)\left(T_{0}+T_{1}\right)+\sum_{i=1}^{n} K\left(I_{i}^{2}\right)\right)\|w-z\|_{T_{1}} .
\end{aligned}
$$

Similarly,

$$
\begin{aligned}
\left\|\Phi_{2}(u, w)-\Phi_{2}(v, z)\right\| \leq & \left(\|C\|_{T_{1}} K^{1}(f)\left(T_{0}+T_{1}\right)+\|B\|_{T_{1}} \sum_{i=1}^{n} K\left(F_{i}\right)\right)\|u-v\|_{T_{1}} \\
& +\|C\|_{T_{1}}\left(K^{2}(f)\left(T_{0}+T_{1}\right)+\sum_{i=1}^{n} K\left(I_{i}^{2}\right)\right)\|w-z\|_{T_{1}} .
\end{aligned}
$$

Inequalities (2.8) and (2.9) and the assumption $\max \left\{\Lambda_{1}, \Lambda_{2}\right\}<1$ imply that $\Phi$ is a contraction. Clearly, the unique fixed point of $\Phi$, is the unique mild solution of (1.1). The proof is completed.

COROLLARY 2.4. Let assumptions (A1) and (A2) be satisfied and assume that $I_{i}^{1} \equiv 0$ for $i=1,2, \ldots, n$. If $\max \left\{\Lambda_{1}, \Lambda_{2}\right\}<1$, where

$$
\begin{aligned}
& \Lambda_{1}=\left(\|S\|_{T_{1}}+\|C\|_{T_{1}}\right) K^{1}(f)\left(T_{0}+T_{1}\right), \\
& \Lambda_{2}=\left(\|S\|_{T_{1}}+\|C\|_{T_{1}}\right)\left(K^{2}(f)\left(T_{0}+T_{1}\right)+\sum_{i=1}^{n} K\left(I_{i}^{2}\right)\right),
\end{aligned}
$$

then there exists a unique mild solution, $u(\cdot)$, of problem (1.1). Moreover $u(\cdot)$ is continuous. 
Now we consider, briefly, some simple examples of functions satisfying assumption (A3).

(1) If $[D(A)]$ is the space $D(A)$ endowed with the graph norm, see (1.5), and $I: X \rightarrow$ $[D(A)]$ is Lipschitz continuous, then $(d / d t) C(t) I(x)=A S(t) I(x)=S(t) A I(x)$ for each $x \in X$.

(2) The cosine family $(C(t))_{t \in \mathbb{R}}$ verifies the condition $F$ (see Travis [10] for details), if there exist a closed operator $B: D(B) \rightarrow X$ such $B^{2}=A$ and $B S(\cdot)$ is a strongly continuous family of bounded linear operators. If $[D(B)]$ is the space $D(B)$ provided with the graph norm, see (1.5), and the function $I: X \rightarrow[D(B)]$ is Lipschitz continuous, then $(d / d t) C(t) I(x)=A S(t) I(x)=B^{2} S(t) I(x)=B S(t) B I(x)$ for every $x \in X$.

(3) Let $r \in \mathbb{R}$ and $g: X \rightarrow X$ a Lipschitz function. If $I(x)=S(r) g(x)$, from Travis [10, Proposition 2.2], $(d / d t) C(t) I(x)=A S(t) S(r) g(x)=(1 / 2)(C(r+t)-C(r-t)) g(x)$, for each $x \in X$.

2.2. Classical solutions. Next, we establish the existence of classical solution for the impulsive initial value problem (1.1), under the assumption that $f$ is continuously differentiable. For this purpose, we need the following lemmas.

LEMMA 2.5. If $g \in C([0, b]: X) \cap C_{b}^{1}((0, b): X), b>0$, then $h(t)=\int_{0}^{t} C(t-s) g(s) d s$ $\in E$ for every $t \in[0, b]$.

Proof. Let $t \in[0, b]$. Since $g$ is continuously differentiable

$$
\begin{aligned}
\int_{0}^{t} C(t-s) g(s) d s & =\int_{0}^{t} C(t-s) g(0) d s+\int_{0}^{t} C(t-s) \int_{0}^{s} g^{\prime}(\mu) d \mu d s \\
& =\int_{0}^{t} C(t-s) g(0) d s+\int_{0}^{t} \int_{0}^{t-\mu} C(\xi) g^{\prime}(\mu) d \xi d \mu,
\end{aligned}
$$

thus

$$
\int_{0}^{t} C(t-s) g(s) d s=S(t) g(0)+\int_{0}^{t} S(t-\mu) g^{\prime}(\mu) d \mu .
$$

We know that $S(t) g(0) \in E$, thus we only need to proof that the second term in the right-hand side of (2.12) is in $E$. In relation with this property, for $\rho \in \mathbb{R}$, we have

$$
\begin{aligned}
\frac{C(\rho+h)-C(\rho)}{h} \int_{0}^{t} S(t-s) g^{\prime}(s) d s= & \frac{1}{2 h} \int_{0}^{t}(S(t-s+\rho+h)-S(t-s+\rho)) g^{\prime}(s) d s \\
& +\frac{1}{2 h} \int_{0}^{t}(S(t-s-\rho-h)-S(t-s-\rho)) g^{\prime}(s) d s,
\end{aligned}
$$

thus

$$
\frac{d}{d \rho} C(\rho) \int_{0}^{t} S(t-s) g^{\prime}(s) d s=\frac{1}{2} \int_{0}^{t}(C(t-s+\rho)-C(t-s-\rho)) g^{\prime}(s) d s .
$$

Clearly, the right-hand side of equation (2.14) is a continuous function of $t$, therefore $\int_{0}^{t} S(t-s) g^{\prime}(s) d s \in E$. The proof is finished. 
LEMMA 2.6. Let $\tilde{x}_{0} \in D(A), \tilde{y}_{0} \in E$ and assume that the assumption in Theorem 2.3 holds. Suppose, furthermore, that $f$ is continuously differentiable and that there exists $K^{3}(f)>0$ such that

$$
\max \left\{\left\|D_{j} f(t, x, y)-D_{j} f(t, x, \tilde{y})\right\|: j=1,2,3\right\} \leq K^{3}(f)\|y-\tilde{y}\|
$$

for every $(t, x, y),(t, x, \tilde{y}) \in\left[-T_{0}, T_{1}\right] \times X^{2}$.

If $t_{i}$ and $t_{i+1}$, are two consecutive impulse instants, then there exists a unique classical solution $u(\cdot)$ of

$$
\begin{gathered}
\ddot{x}(t)=A x(t)+f(t, x(t), \dot{x}(t)), \quad t \in\left(-T_{0}, t_{i+1}\right), \\
x\left(t_{i}\right)=\tilde{x}_{0}, \quad \dot{x}\left(t_{i}\right)=\tilde{y}_{0} .
\end{gathered}
$$

Moreover, $u\left(t_{i+1}^{-}\right) \in D(A)$ and $\dot{u}\left(t_{i+1}^{-}\right) \in E$.

Proof. Let $w:\left(-T_{0}, T_{1}\right) \rightarrow X$ be the unique mild solution of

$$
\begin{gathered}
\ddot{x}(t)=A x(t)+f(t, x(t), \dot{x}(t)), \quad t \in\left(-T_{0}, T_{1}\right), \\
x\left(t_{i}\right)=\tilde{x}_{0}, \quad \dot{x}\left(t_{i}\right)=\tilde{y}_{0},
\end{gathered}
$$

and let $u:\left(-T_{0}, t_{i+1}\right) \rightarrow X$ be the mild solution of the initial value problem (2.16). From the proof of [10, Proposition 3.3], we infer that $w$ and $u$ are classical solutions and thus, that $u\left(t_{i+1}\right)=w\left(t_{i+1}\right) \in D(A)$, since $u=w$ on $\left(-T_{0}, t_{i+1}\right)$.

On the other hand,

$$
\begin{aligned}
\dot{u}\left(t_{i+1}^{-}\right)= & A S\left(t_{i+1}\right) \tilde{x}_{0}+C\left(t_{i+1}\right) \tilde{y}_{0} \\
& +\int_{0}^{t_{i+1}} C\left(t_{i+1}-s\right) f(s, u(s), \dot{u}(s)) d s \\
= & \dot{w}\left(t_{i+1}\right) .
\end{aligned}
$$

Clearly, $A S\left(t_{i+1}\right) \tilde{x}_{0}+C\left(t_{i+1}\right) \tilde{y}_{0} \in E$ and from Lemma 2.5, the integral term in (2.18) is in $E$, therefore $\dot{w}\left(t_{i+1}\right) \in E$. The proof is completed.

Now we establish the principal result of this paper.

THEOREM 2.7 (classical solution). Let $x_{0} \in D(A), y_{0} \in E$ and assume that the assumptions in Theorem 2.3 and Lemma 2.6 hold. If $(u, v):\left(-T_{0}, T_{1}\right) \rightarrow X$ is the mild solution of (1.1) and $I_{i}^{1}\left(u\left(t_{i}\right)\right) \in D(A), I_{i}^{2}\left(\dot{u}\left(t_{i}^{+}\right)\right) \in E$ for each $i \in\{1,2, \ldots, n\}$, then $(u, v)$ is a classical solution.

Proof. Let $w:\left(-T_{0}, t_{1}\right) \rightarrow X$ be the unique classical solution of

$$
\begin{gathered}
\ddot{x}(t)=A x(t)+f(t, x(t), \dot{x}(t)), \quad t \in\left(-T_{0}, t_{1}\right), \\
x(0)=x_{0}, \quad \dot{x}(0)=y_{0}
\end{gathered}
$$


and let $\left(u_{1}, v_{1}\right):\left(-T_{0}, t_{1}\right] \rightarrow X \times X$ be the function defined by $\left(u_{1}, v_{1}\right)(t)=\left(w\left(t^{-}\right)\right.$, $\left.\dot{w}\left(t^{-}\right)\right)$. From Lemma 2.6, we know that $\left(u_{1}\left(t_{1}\right), v_{1}\left(t_{1}\right)\right) \in D(A) \times E$; thus, there exists a unique classical solution $w(\cdot)$ of the abstract Cauchy problem

$$
\begin{gathered}
\ddot{x}(t)=A x(t)+f(t, x(t), \dot{x}(t)), \quad t \in\left(-T_{0}, t_{2}\right), \\
x\left(t_{1}\right)=u_{1}\left(t_{1}\right)+I_{1}^{1}\left(u\left(t_{1}\right)\right), \quad \dot{x}\left(t_{1}\right)=v_{1}\left(t_{1}\right)+I_{1}^{2}\left(\dot{u}\left(t_{1}^{+}\right)\right) .
\end{gathered}
$$

Similarly to the previous case, $\left(u_{2}, v_{2}\right):\left(-T_{0}, t_{2}\right] \rightarrow X \times X$ will be the function defined by $\left(u_{2}, v_{2}\right)(t)=\left(w\left(t^{-}\right), \dot{w}\left(t^{-}\right)\right)$.

In general, if $w$ is the classical solution of the second-order Cauchy problem

$$
\begin{gathered}
\ddot{x}(t)=A x(t)+f(t, x(t), \dot{x}(t)), \quad t \in\left(-T_{0}, t_{k}\right), \\
x\left(t_{k-1}\right)=u_{k-1}\left(t_{k-1}\right)+I_{k-1}^{1}\left(u\left(t_{k-1}\right)\right), \\
\dot{x}\left(t_{k-1}\right)=v_{k-1}\left(t_{k-1}\right)+I_{k-1}^{2}\left(\dot{u}\left(t_{k-1}^{+}\right)\right),
\end{gathered}
$$

we denote by $\left(u_{k}, v_{k}\right)$ the function $\left(u_{k}, v_{k}\right):\left(-T_{0}, t_{k}\right] \rightarrow X$, where $u_{k}(t)=w\left(t^{-}\right)$and $v_{k}(t)=\dot{w}\left(t^{-}\right)$.

Let $(\tilde{u}, \tilde{v}):\left(-T_{0}, T_{1}\right) \rightarrow X$ be the function defined by

$$
(\tilde{u}(t), \tilde{v}(t))= \begin{cases}\left(u_{1}(t), v_{1}(t)\right) & \text { if }-T_{0}<t \leq t_{1} \\ \left(u_{k}(t), v_{k}(t)\right) & \text { if } t_{k-1}<t \leq t_{k} \\ \left(u_{n+1}(t), v_{n+1}(t)\right) & \text { if } t_{n}<t<T_{1}\end{cases}
$$

It is easy to see that $(\tilde{u}, \tilde{v})$ is the unique classical solution of the impulsive problem

$$
\begin{gathered}
\ddot{x}(t)=A x(t)+f(t, x(t), \dot{x}(t)), \quad t \in\left(-T_{0}, T_{1}\right), t \neq t_{i}, \\
x(0)=x_{0}, \quad \dot{x}(0)=y_{0}, \\
\triangle x\left(t_{i}\right)=I_{i}^{1}\left(u\left(t_{i}\right)\right), \quad \triangle \dot{x}\left(t_{i}\right)=I_{i}^{2}\left(\dot{u}\left(t_{i}^{+}\right)\right) .
\end{gathered}
$$

Next, we show that $(\tilde{u}, \tilde{v})=(u, v)$. In order to reduce the proof, we introduce the group of linear operators

$$
U(t)=\left(\begin{array}{cc}
C(t) & S(t) \\
A S(t) & C(t)
\end{array}\right)_{t \in \mathbb{R}}
$$

on $D(A) \times E$. The function $(\tilde{u}, \tilde{v})$ is a solution of the first-order impulsive Cauchy problem

$$
\begin{gathered}
\dot{W}(t)=\left(\begin{array}{ll}
0 & I \\
A & 0
\end{array}\right) W(t)+F(t, W(t)), \\
W(0)=\left(\begin{array}{l}
x_{0} \\
y_{0}
\end{array}\right), \quad \triangle W\left(t_{i}\right)=I_{i}\left(\begin{array}{c}
u\left(t_{i}\right) \\
\dot{u}\left(t_{i}^{+}\right)
\end{array}\right),
\end{gathered}
$$


where $I_{i}$ and $F$ are defined in obvious manner. From the proof of [5, Lemma 2.3], we infer that

$$
W(t)=U(t)\left(\begin{array}{l}
x_{0} \\
y_{0}
\end{array}\right)+\int_{0}^{t} U(t-s) F(s, W(s)) d s+\sum_{t_{i}<t} U\left(t-t_{i}\right) I_{i}\left(\begin{array}{c}
u\left(t_{i}\right) \\
\dot{u}\left(t_{i}^{+}\right)
\end{array}\right),
$$

thus

$$
\begin{aligned}
\tilde{u}(t)= & C(t) x_{0}+S(t) y_{0}+\int_{0}^{t} S(t-s) f(s, \tilde{u}(s), \tilde{v}(s)) d s \\
& +\sum_{0<t_{i}<t} C\left(t-t_{i}\right) I_{i}^{1}\left(u\left(t_{i}\right)\right)+\sum_{0<t_{i}<t} S\left(t-t_{i}\right) I_{i}^{2}\left(\dot{u}\left(t_{i}^{+}\right)\right), \\
\tilde{v}(t)= & A S(t) x_{0}+C(t) y_{0}+\int_{0}^{t} C(t-s) f(s, \tilde{u}(s), \tilde{v}(s)) d s \\
& +\sum_{0<t_{i}<t} A S\left(t-t_{i}\right) I_{i}^{1}\left(u\left(t_{i}\right)\right)+\sum_{0<t_{i}<t} C\left(t-t_{i}\right) I_{i}^{2}\left(\dot{u}\left(t_{i}^{+}\right)\right) .
\end{aligned}
$$

Finally, using $\theta(t)=\max \{\|u(t)-\tilde{u}(t)\|,\|v(t)-\tilde{v}(t)\|\}$, from assumption (A1) and (2.27), we obtain that

$$
\theta(t) \leq C_{1} \int_{0}^{t} \theta(s) d s,
$$

where $C_{1}$ is a constant independent of $t \in\left(-T_{0}, T_{1}\right)$. The Gronwall's inequality implies that $u=\tilde{u}$. The proof is completed.

3. Consequences. Next, we briefly consider the impulsive system

$$
\begin{gathered}
\ddot{u}(t)=A u(t)+f(t, u(t)), \quad t \in\left(-T_{0}, T_{1}\right), \\
u(0)=x_{0}, \quad \dot{u}(0)=y_{0}, \\
\triangle u\left(t_{i}\right)=I_{i}^{1}\left(u\left(t_{i}\right)\right), \quad \triangle \dot{u}\left(t_{i}\right)=I_{i}^{2}\left(u\left(t_{i}\right)\right) .
\end{gathered}
$$

DEFINITION 3.1. A function $u \in P C_{b}\left(\left(-T_{0}, T_{1}\right): X\right)$ is a mild solution of the secondorder impulsive problem (3.1) if

$$
\begin{aligned}
u(t)= & C(t) x_{0}+S(t) y_{0}+\int_{0}^{t} S(t-s) f(s, u(s)) d s \\
& +\sum_{0<t_{i}<t} C\left(t-t_{i}\right) I_{i}^{1}\left(u\left(t_{i}\right)\right)+\sum_{0<t_{i}<t} S\left(t-t_{i}\right) I_{i}^{2}\left(u\left(t_{i}\right)\right)
\end{aligned}
$$

for each $t \in\left(-T_{0}, T_{1}\right)$.

DEFINITION 3.2. A function $u:\left(-T_{0}, T_{1}\right) \rightarrow X$ is a classical solution of the impulsive problem (3.1) if $u \in P C_{b}\left(\left(-T_{0}, T_{1}\right): X\right) \cap C^{2}\left(\left(-T_{0}, T_{1}\right) \backslash\left\{t_{1}, t_{2}, \ldots, t_{n}\right\}: X\right)$ and (3.1) is satisfied.

In order to establish our next result, we introduce one more simple assumption:

(A4) the functions $f: \mathbb{R} \times X \rightarrow X, I_{i}^{j}: X \rightarrow X$ are continuous and exist positive constants $K(f)$ and $K\left(I_{i}^{1}\right)$ such that

$$
\begin{gathered}
\left\|f(t, u)-f\left(t, u^{\prime}\right)\right\| \leq K^{1}(f)\left\|u-u^{\prime}\right\|, \\
\left\|I_{i}^{j}(u)-I_{i}^{j}\left(u^{\prime}\right)\right\| \leq K\left(I_{i}^{j}\right)\left\|u-u^{\prime}\right\|,
\end{gathered}
$$

for every $u, u^{\prime} \in X$. 
THEOREM 3.3. Let $x_{0}, y_{0} \in X$ and assume that assumptions (A2), (A4) hold. If

$$
\|S\|_{T_{1}} K^{1}(f)\left(T_{0}+T_{1}\right)+\sum_{i=1}^{n}\left(\|C\|_{T_{1}} K\left(I_{i}^{1}\right)+\|S\|_{T_{1}} K\left(I_{i}^{2}\right)\right)<1,
$$

then there exists a unique mild solution of (3.1).

Proof. The proof of this theorem is similar to the proof of Theorem 2.3. We omit the details.

Using the ideas in the proofs of [10, Lemma 3 and Proposition 2.4], we can prove the following lemma.

LEMMA 3.4. Let $\tilde{x}_{0} \in D(A), \tilde{y}_{0} \in E$, and assume that the hypotheses in Theorem 3.3 hold. If $f$ is continuously differentiable, then the mild solution, $u(\cdot)$, of

$$
\begin{gathered}
\ddot{x}(t)=A x(t)+f(t, x(t)), \quad t \in\left(-T_{0}, t_{i+1}\right), \\
x\left(t_{i}\right)=\tilde{x}_{0}, \quad \dot{x}\left(t_{i}\right)=\tilde{y}_{0},
\end{gathered}
$$

is a classical solution. Moreover, $u\left(t_{i+1}^{-}\right) \in D(A)$ and $\dot{u}\left(t_{i+1}^{-}\right) \in E$.

The proof of Theorem 3.5 follows from the steps in the proof of Theorem 2.7.

THEOREM 3.5 (classical solution). Let $x_{0} \in D(A), y_{0} \in E$, and assume that the assumptions in Theorem 3.3 and Lemma 3.4 hold. If $u:\left(-T_{0}, T_{1}\right) \rightarrow X$ is the mild solution of the impulsive problem (3.1) and $I_{i}^{1}\left(u\left(t_{i}\right)\right) \in D(A), I_{i}^{2}\left(u\left(t_{i}\right)\right) \in E$ for every $i \in\{1,2, \ldots, n\}$, then $u$ is a classical solution.

ACKNOWLEDGMENT. The author wishes to thank the referee for comments and suggestions.

\section{REFERENCES}

[1] A. Cabada, J. J. Nieto, D. Franco, and S. I. Trofimchuk, A generalization of the monotone method for second order periodic boundary value problem with impulses at fixed points, Dynam. Contin. Discrete Impuls. Systems 7 (2000), no. 1, 145-158.

[2] H. O. Fattorini, Second Order Linear Differential Equations in Banach Spaces, NorthHolland Mathematics Studies. vol. 108., North-Holland Publishing, Amsterdam, 1985.

[3] S. Jinli and M. Yihai, Initial value problems for the second order mixed monotone type of impulsive differential equations in Banach spaces, J. Math. Anal. Appl. 247 (2000), no. 2, 506-516.

[4] J. Kisyński, On a second order Cauchy's problem in a Banach space, Bull. Acad. Polon. Sci. Sér. Sci. Math. Astronom. Phys. 18 (1970), 371-374.

[5] J. H. Liu, Nonlinear impulsive evolution equations, Dynam. Contin. Discrete Impuls. Systems 6 (1999), no. 1, 77-85.

[6] Y. V. Rogovchenko, Impulsive evolution systems: main results and new trends, Dynam. Contin. Discrete Impuls. Systems 3 (1997), no. 1, 57-88.

[7] _ Nonlinear impulse evolution systems and applications to population models, J. Math. Anal. Appl. 207 (1997), no. 2, 300-315.

[8] C. C. Travis and G. F. Webb, Compactness, regularity, and uniform continuity properties of strongly continuous cosine families, Houston J. Math. 3 (1977), no. 4, 555-567.

[9] __ Cosine families and abstract nonlinear second order differential equations, Acta Math. Acad. Sci. Hungar. 32 (1978), no. 1-2, 75-96. 
[10] Second order differential equations in Banach space, Nonlinear Equations in Abstract Spaces (Proc. Internat. Sympos., Univ. Texas, Arlington, Tex., 1977), Academic Press, New York, 1978, pp. 331-361.

Eduardo Hernández Morales: Departamento de Matemática, Instituto de Ciências matemáticas de São Carlos, Universidade de São Paulo, Caixa Postal 668, 13560-970 SÃo CARLOS, SÃo PAUlo, BRAZIL

E-mail address: 1a1ohm@icmc.sc.usp.br 


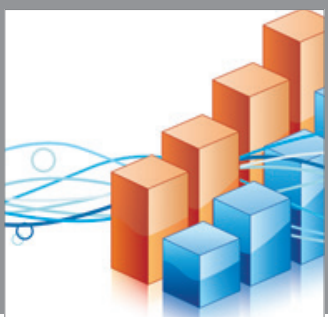

Advances in

Operations Research

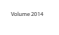

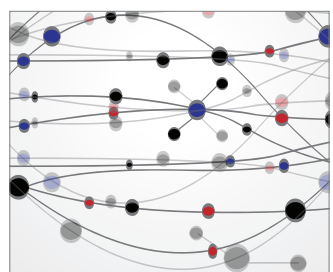

\section{The Scientific} World Journal
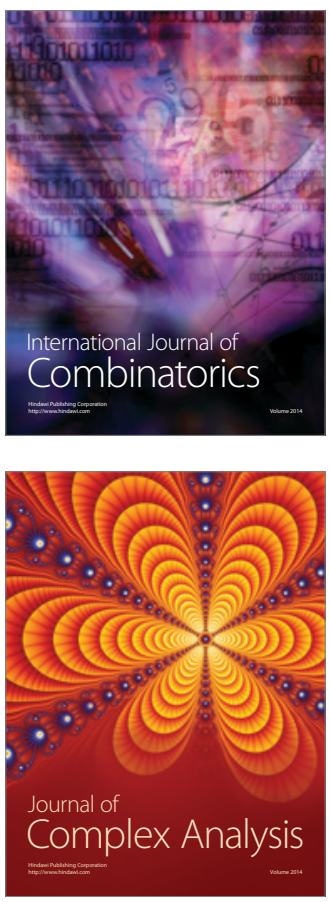

International Journal of

Mathematics and

Mathematical

Sciences
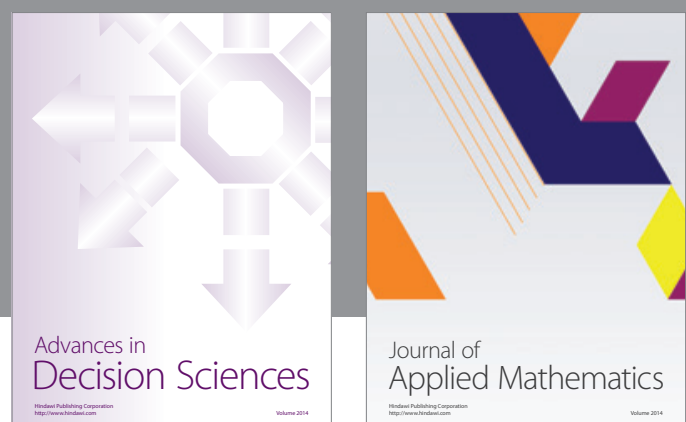

Journal of

Applied Mathematics
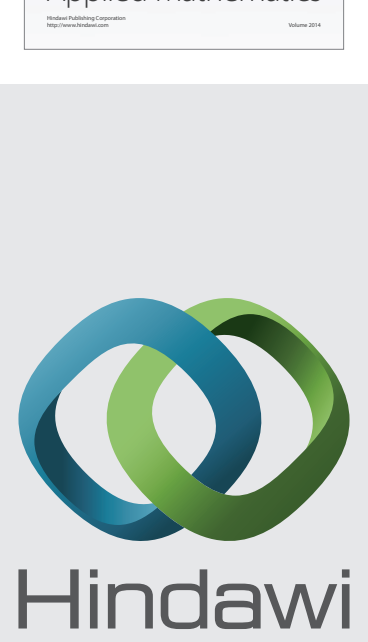

Submit your manuscripts at http://www.hindawi.com
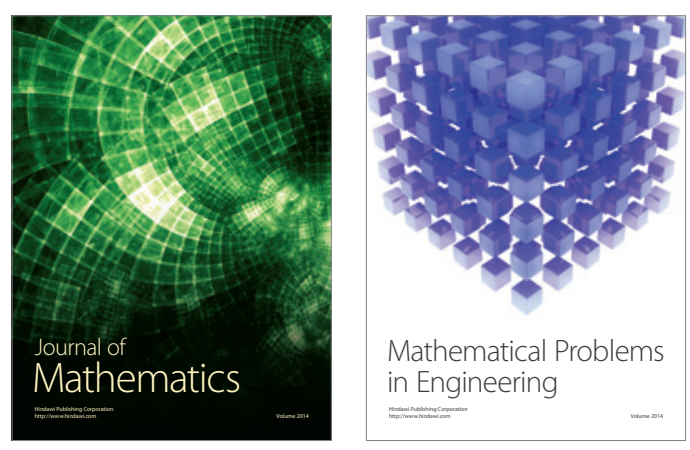

Mathematical Problems in Engineering
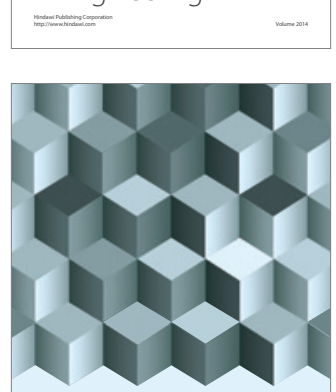

Journal of

Function Spaces
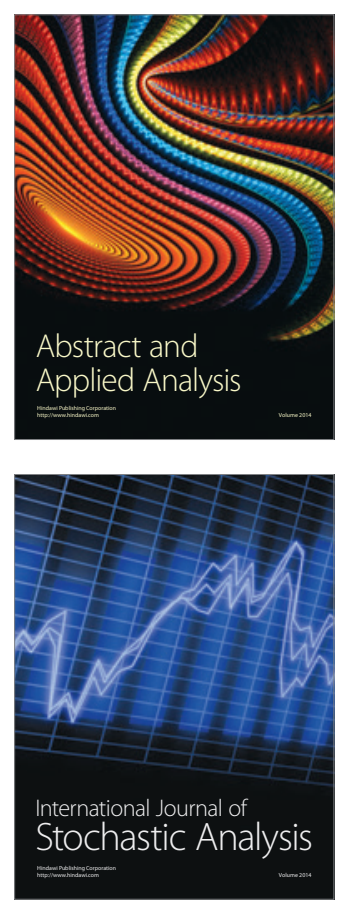

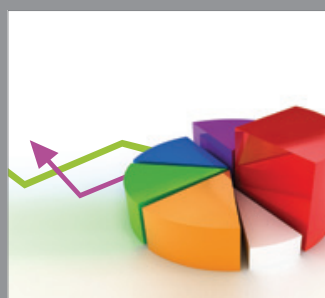

ournal of

Probability and Statistics

Promensencen
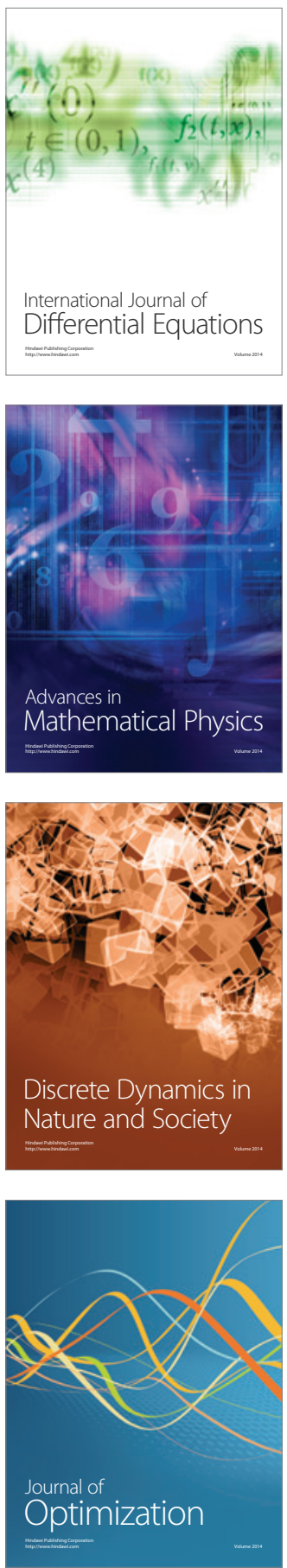